\title{
Exploration of the Hemispheric Differences in Number Processing of the Brain
}

\begin{abstract}
Aaron Wyland Walters ${ }^{\mathrm{a}}$
The current study explored how reaction time and accuracy differed in the left and right visual fields by altering various dot clusters in both number and organization. Researchers have hypothesized that the left hemisphere uses counting to judge small, disorganized clusters of objects accurately and that the right hemisphere uses estimation to judge clusters organized in geometric shape accurately. The current study tested both visual fields of participant's with organized and unorganized clusters of dots. Dots were clustered between 3 and 12. The clusters were presented on separate sides of a computer screen to analyze visual field differences in counting and estimation. A central target was presented on the screen to maintain central focus for the visual fields. Data from 40 participants (30 men, 10 women) from a small liberal arts college indicated that when clusters reached between 7 and 8 dots, organization in the right visual field created inaccuracy in judgment. Reaction time data indicated that as number level increased, reaction time slowed. Reaction time data also showed that organization slowed reaction times in both visual fields. These data indicated that different numerical judgment abilities do exist within the hemispheres.
\end{abstract}

Keywords: hemisphere, dot clusters, visual fields, estimation, counting

\section{Introduction}

Life requires people to make guesses and to think about possible outcomes in relation to specific actions on a daily basis. Many of these uncertain situations that we experience require us to make approximations dealing with numbers, time intervals, weights, or distances. For example, we may have to estimate how many of a certain object we think there may be, or how long we think a task may take. When trying to understand the processes of human calculation and our understanding of numbers, there are two separate processes to consider; estimation and counting. These are the two most basic types of number processing that people use (Nieder \& Dehaene, 2009). Though these two processes have been explored individually, there is no study that has directly compared the similarities and differences of the left and right hemispheres and the processes located in each hemisphere. This study explored how these two different processes affect the numerical estimation and counting abilities of people in relation to the separate hemispheres of the brain and their specific functions.

Counting is a process that is very straightforward when trying to understand the way humans translate numerical information. The process of counting is located in the right visual field and is ultimately controlled by the left hemisphere. Counting models (Nieder \& Dehaene, 2009) suggest that when we count we take into consideration three parts of the items being observed. The first part of counting that we observe is noticing the space that all of the individual objects under observation occupy. This allows each object to be counted separately. Without this ability we would group items together and be unable to discriminate the separation between individual items. The second part of counting is the ability to keep track of objects that have already been counted so that items are not counted again. This aspect is essential in getting an accurate count of items because without this ability we would lack the capability to remember which items have been counted previously and which have not yet been counted. The last part of counting that is observed in human beings is the ability to update the total number of items mentally that had been previously counted. We have the ability to keep an internal conversation with ourselves so that information concerning a total number of items we have counted to a certain point is not forgotten when the next item is observed and stored with the new total. Without this internal conversation we would be unable to process information not only in the aspect of numbers but also in many other aspects of our daily lives. These three concepts together make up the process that allows human beings to have the ability to count accurately (Nieder \& Dehaene, 2009). Though counting processes have been tested before to indicate which hemisphere the counting ability lies in, this process has never been studied in an experiment that compares both counting and estimation in both the left and right hemispheres. My study will compare the similarities and differences between the left and right hemispheres and the processes of counting and estimation. The process of estimation is a little more advanced than that of counting, however.

Unlike the more clearly defined counting models described in the previous paragraph, estimation models indicate that there is an internal system that allows for us to make a reasonable guess when viewing larger numbers or clusters of objects (Piazza, Mechelli, Price, \& Butterworth, 2006). The process of estimation is located in the left visual field and is ultimately controlled by the right hemisphere. According to this model, objects that are being estimated by an individual undergo a process by which the type of stimuli being estimated are matched with a sensory input area (seeing, hearing, feeling, tasting, touching). This process allows us to relate the item being estimated to a specific area of the body. For instance, if we are trying to guess how many dogs we hear barking, we focus our attention to listen to the dogs barking and try to separate how many different barks we believe to hear. Another part of this analysis procedure is the ability to gauge whether the objects being analyzed are joined 
together or separate entities indicating that number shifts are apparent in every sensory area of the body. During this brief analysis, the mind makes an assessment of all the different information that is taken into consideration and a number value is produced.

Each individual has his/her own system of number judgment. Judgment can vary from very poor to very accurate based on the sensory data that the brain uses during the short amount of time that it has to analyze specific objects. The estimation performance in human beings and animals is on average a reasonable guess, but as the number of objects that are being estimated increases, the more the accuracy of estimation decreases (Piazza \& Dehaene, 2004). This experiment will look directly at the relationship between how an increased or decreased amount of stimuli can affect both the counting and estimation processes of each participant.

Many different stimuli can play a role in either helping or hurting estimation abilities. Koesling, Carbone, Pomplun, Sichelschmidt, and Ritter (2004) explored these stimuli in their study that contained several examples of the different types of inputs that can influence the estimation process. The dimensions of the objects being observed can have a considerable impact on estimation. It was shown that with increasing size of the objects came overestimation of the total number of objects. A second aspect of this study that was important in number processing was the spacing between the objects that were being estimated. The accuracy of number estimation appeared to change as the spacing between the objects increased. This concept led the authors to the conclusion that people appeared to overestimate as the space that the objects occupied increased (Koesling et al., 2004). Though this study had many valuable conclusions on how different stimuli can affect the estimation processes, it lacked the same conclusive data to show any information about how stimuli can affect the counting process. The current study will compare both estimation and counting processes and how these different variables can have an effect on the processing of visual numerical stimuli. The same stimuli (varied dot clusters) will be presented to the participants in each of their visual fields so that a clear recording can be measured on which hemisphere has a greater or lesser activation in estimation or counting, and which stimuli will have the greatest positive or negative effect on each hemisphere and the individual numerical processes that each possess.

Recent studies have begun to explore which specific parts of the brain are responsible for the two techniques that we use for number calculation and how these parts of the brain affect the ways objects are processed numerically. A recent fMRI (functional magnetic resonance imaging) study demonstrated that the processes of arithmetic and other number operations that take time and consideration to solve reside in the left hemisphere of the brain (Nieder \& Dehaene, 2009). Though the fMRI study has contributed a great amount of data towards where the processes of estimation and counting lie specifically in the brain, the test does not take into consideration the time that the fMRI participants take to solve these arithmetic problems. Time is directly related to the processes that our brain (both left and right hemispheres) computes on a day-to-day basis. The current study will take reaction time into account by ensuring that participants will only have a limited time to view the stimuli (clusters of dots) so that estimation and counting processes can be clearly separated. If the viewing time is not closely monitored in a study, participants will take as much time as they can to use their counting abilities in comparison to their estimation abilities because counting abilities are far more accurate than estimation abilities. By using one steady time to allow for the viewing of the stimuli, participants will be forced to use counting abilities for smaller clusters of dots and their estimation abilities for larger clusters of dots.

A different fMRI study indicated very similar patterns of left hemispheric activation when participants dealt with counting in comparison to estimation (Piazza et al., 2006). One observation of this study that differed from the Nieder \& Dehaene (2009) study was that left hemispheric activation was recorded while participants were completing estimation tasks. While participants were completing counting tasks it was also discovered that the right hemisphere was active. Though this seems to disagree with the initial theory of the left hemisphere being related to counting and the right hemisphere being related to estimation, activation of the opposite hemispheres was only minimal in the tasks. There was still an overwhelming amount of activity recorded in the left hemisphere when counting tasks were present, and the same was recorded during right hemisphere activation when dealing with estimation (Piazza et al., 2006). Again, this fMRI study failed to take into consideration the variable of reaction time in the participants. In the current experiment, reaction time of participants to certain stimuli consisting of both large and small clusters of dots will be measured. It is predicted that larger dot clusters will yield a slower reaction time and smaller dot clusters will yield a faster reaction time when viewed by participants.

In the present study, estimation and counting differences will be measured in relation to both the left and right hemispheres of the brain using the visual stimuli of dot clusters that will be presented on a computer screen. These stimuli will target both the left and right hemispheres individually and will allow for measuring the differences between both the left and right hemispheres based on reaction time and accuracy to the stimuli presented. From previous research, it is predicted that the right hemisphere will be more accurate in estimating larger clusters that are organized in a geometric shape of some form. This result would support that the organization of shapes allows the right hemisphere to categorize items more quickly and efficiently (Piazza, Mechelli, Price, \& Butterworth, 2006). It is also predicted from past research that the left hemisphere will be accurate in counting clusters that are of smaller quantity and that can either be organized or unorganized. This would indicate that the left hemisphere will use its counting system to add the items while also being able to keep a mentally tabulated count (Nieder \& Dehaene, 2009).

\section{Method}

Design

A 2 (structure) x 2 (visual field) x 5 (quantity of dots) repeated measures design was used in which structure was either a geometric organization of dots or an unorganized cluster of dots, visual field was presentation of the dots to the left or right visual field and the quantity of dots compared were 3 to 4,5 to 6,7 to 8,9 to 10 , and 11 to 12 . 


\section{Participants}

This study used 40 participants ( 10 women, 30 men) who all completed the same randomized task. All the participants were undergraduate students who attended Westminster College and who were all at least 18 years of age. The students who participated in the current experiment had no previous knowledge of the test. Only right handed participants were used in the experiment. The current experiment followed all ethical guidelines (American Psychological Association). Participants received no incentive for participation in my experiment unless they were enrolled in an introduction to psychology course in which they were required to participate in the experiment for course credit.

\section{Example Of Single Program Trial}

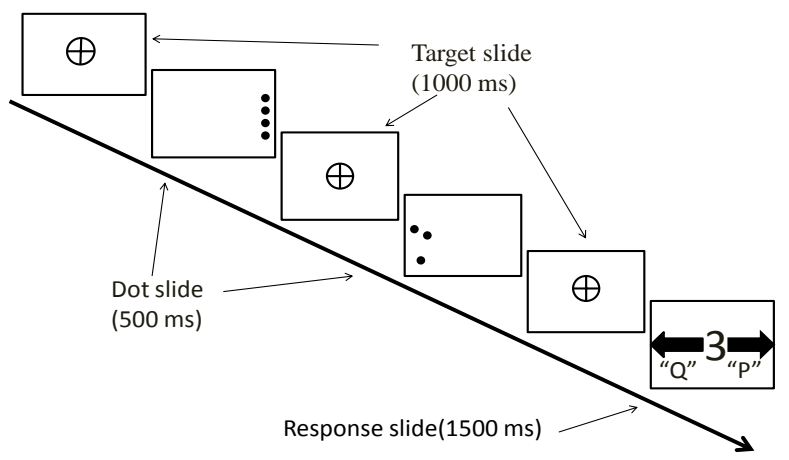

Figure 1: This figure is a representation of one trial during the experiment. The progression is shown beginning from the top left of the picture to the bottom right of the picture taking into account the display time in between each presentation as described in the figure.

\section{Apparatus}

The apparatus that was used to conduct the experiment was a Dell computer with a Pentium processor that allowed the images of dot clusters to be displayed that were used in the experiment. The program that was used to create the dot cluster slide show and also used for data collection was EPrime. The program that was designed displayed dot images to the participants. The computer screen was approximately $171 / 2$ inches in size with a screen resolution of $1920 \times 1080$. The images that appeared on the screen were identical in size along with each dot being identical in size (400 pixels x 400 pixels).

\section{Procedure}

The participant was first presented with an informed consent form. After being seated at the computer, they were instructed to keep their eyes focused on the center of the screen in order to maintain the efficiency of testing the differences in the left and right hemispheres. The distance from the participant's eyes to the center of the screen was also measured in order to maintain a constant distance across trials so that visual abilities would be the same across all participants. The distance from each participant to the screen was $57 \mathrm{~cm}$ based on the paradigm that was used (Ratinckx, Brysbaert, \& Reynvoet, 2001). A target was also added to a slide and presented in the center of the screen between dot slides to help maintain the focus of the participant. Initially, the participant was shown a target slide for $1000 \mathrm{~ms}$ in order to maintain the central focus of both visual fields which was very important to this study so that brain lateralization could accurately be measured. The participant was then shown one image in the right field of vision with a number of dots between 3 and 12 in either a random organization or a geometric organization. The pattern and number of dots allowed for the measuring of the estimation ability of the left visual field and the counting ability of the right visual field because each responded differently to these variables. After one dot slide was shown in the right visual field for $500 \mathrm{~ms}$, a target slide was then shown to the participant for approximately $1000 \mathrm{~ms}$. Again, the target slide helped to maintain the focus of both fields of vision in the middle of the screen before the following dot slide presentation. The amount of time that participants had to observe the individual slides had a significant effect on the estimation and counting abilities. The longer the amount of time that was allowed for viewing the dot slides, the more counting was used, the shorter the amount of time allotted, the more estimation ability was used. After the target slide was shown, the second dot slide was shown in the left field of vision with the number of dots differing by either plus one or minus one from the first dot slide and was presented in the opposite organization style from the first dot slide. The difference of one dot between dot slides was to show how close numbers represented the different numerical processes located in each visual field based on the counting and estimation properties of the hemispheres of the brain. For instance, if the group of dots presented in the right visual field contained five dots in an organized cluster, then the group of dots presented in the left visual field would contain six dots in an unorganized cluster. The dot slides varied by only one dot per trial so that the presentations would be similar in size and could effectively measure the differences between hemispheric numerical ability. It was expected that clusters of organized dots would be estimated more efficiently by the right hemisphere (LVF) and smaller clusters of unorganized dots would be counted more efficiently by the left hemisphere (RVF).

Number level in the experiment was organized into five number levels. Number level one compared clusters between 3 and 4 dots, number level two compared clusters between 5 and 6 dots, number level three compared clusters between 7 and 8 dots, number level four compared clusters between 9 and 10 dots, and number level five compared clusters between 11 and 12 dots. The second dot slide presentation was opposite in the organization of the first dot slide presentation to test the differences in the processes of the hemispheres within each trial. So if the dots were organized in a set of two columns ( 8 dots) in the first dot slide presentation, they would be scattered randomly $(7$ dots) in the second dot slide presentation. This was crucial to the experiment because it was shown through previous research that the organizational appearance of dots has an effect on the estimation and counting abilities of participants (Piazza \& Dehaene, 2004). The more organized the dot structures, the more estimation ability was used. The more scattered the organization of the dots was, the more the counting process was used.

After the second dot slide was presented then another target slide appeared to the participant for $1000 \mathrm{~ms}$. After the target slide was presented, a response slide was presented in 
the middle of the screen for $1500 \mathrm{~ms}$. The response slide contained two arrows pointing to both sides of the screen that the dots were presented in and a number in the center of the two arrows. The number correlated to the number of dots that was presented in one of the visual fields. The participant's goal was to accurately select which visual field contained the number located in the center of the response slide. This was accomplished by the participant selecting "P" if they believed the number of dots was located in the right visual field and "Q" if they believed the number of dots was located in the left visual field. The response slide was displayed in order for the independent variables of the study to be measured and to compare the differences between the organizations, number, and which hemispheres responded accurately to these variables.

If the participant failed to choose an arrow key within $1500 \mathrm{~ms}$ of the response slide being presented, that particular slide was excluded from the data for response time. If the participant chose an arrow key within $150 \mathrm{~ms}$ or less, the data from those trials was also excluded from the correct response time analysis. This removal of response time was based on the paradigm replicated in the current study.

The slide presentation process was repeated for 120 trials per participant with a total of 40 participants. The order of presentation of the dot slides changed for each participant so that no two participants viewed the 120 trials in the same manner. Participants were orally debriefed at the conclusion of the experiment.

\section{Results}

Analyses focused on participants' reaction times and accuracies for each of the 120 trials of dot clusters. All trials were counterbalanced in organization (organized or scattered) and number level (number levels 1, 2, 3, 4, and 5). The procedure used to analyze the data was adapted from the previous paradigm (Ratinckx, Brysbaert, \& Reynvoet, 2001). Based on the previous paradigm stated, no accuracy data was filtered from the analysis. By measuring accuracy in the data, it was possible to determine which number level $(1=$ dot clusters of 3 and 4, 2= dot clusters of 5 and $6,3=$ dot clusters of 7 and 8, 4= dot clusters of 9 and 10, 5= dot clusters of 11 and 12), and visual field (organized clusters in left or right) had the greatest impact on judgment of dot cluster presentations. Measuring the data in this manner leads to understand the number level and organization that yielded the most efficient counting and estimation in the right and left visual fields respectively. First, accuracy was measured using a repeated measures analysis for visual field, organization, and number level $(2 \times 2 \times 5)$. This uncovered the relationship between the left visual field and organized structure estimation, and the right visual field and small-disorganized cluster counting. A significant 3-way interaction was found between visual field, organization, and number level.

\section{Accuracy (Number Level x Organization x Visual Field)}

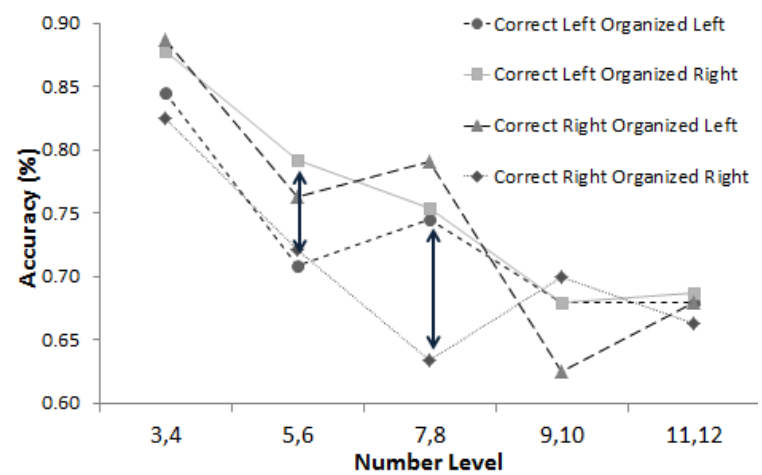

Figure 2: Repeated Measures 3-way Interaction (Visual Field x Organization x Number Level)

Figure 2 shows the average accuracy between each of the five number levels, between each type of organization, and between each visual field. (Ratinckx, Brysbaert, \& Reynvoet, 2001). The first two letters of each legend entry simply represent which visual field the correct slide was located in; either the correct slide was in the left visual field (CL) or the right visual field (CR). Organized Left and Organized Right represent which visual field contained the dot cluster that was organized. The arrows in the graph represent the significant comparisons measured in the Organized $\mathrm{x}$ Visual Field $\mathrm{x}$ Number Level analysis, $F(4,36)=3,235, . p=.02, \eta^{2}=.077$. (See Table 2.1 in the appendix for the means and standard deviations)

According to the data analyzed in Figure 2, the threeway interaction was significant. Within the significant 3-way interaction two sets of data points were found to be significant. One area of significance was found in number level two. There was a significant separation between Correct Left Organized Right and Correct Left Organized Left $F$ (1, 39) $=6.440, . p=.02, \eta^{2}=.142$ in which Correct Left Organized Right produced a much higher average accuracy than Correct Left Organized Left. The second number level interaction (dot clusters of 5 and 6 dots) indicated that when participants responded to disorganized dot clusters in the left visual field, a higher average accuracy was achieved than when participants responded to organized dot clusters in the left visual field. This interaction did not support the hypothesis that organization in the left visual field would produce a higher accuracy than organization in the right visual field because of the estimation ability of the right hemisphere. Another significant relationship was found in number level three of the accuracy data. The significance was between Correct Left Organized Left and Correct Right Organized Right, $F(1,39)=8.980, . p=.01, \eta^{2}=.187$. Correct Left Organized Left had a significantly higher average accuracy than Correct Right Organized Right. The third number level interaction (dot clusters of 7 and 8 dots) indicated that when participants responded to organized clusters of dots in the left visual field, a higher average accuracy was achieved than when participants responded to organized clusters of dots in the right visual field. The interaction of number level three did support the hypothesis that organized clusters in the left visual field would be more 
accurately estimated than would counting organized clusters of dots in the right visual field.

All average reaction times with correct responses from participants were analyzed except those that were less than $150 \mathrm{~ms}$ based on the paradigm from previous research (Ratinckx, Brysbaert, \& Reynvoet, 2001). Only six trials were excluded from the 4800 total trials based on this criterion. Error rate for correct average reaction times did not exceed $26 \%$ per participant which was an acceptable error rate average to include in data analysis based on previous research (Ratinckx, Brysbaert, \& Reynvoet, 2001).

\section{Correct Reaction Time (Visual Field x Organization)}

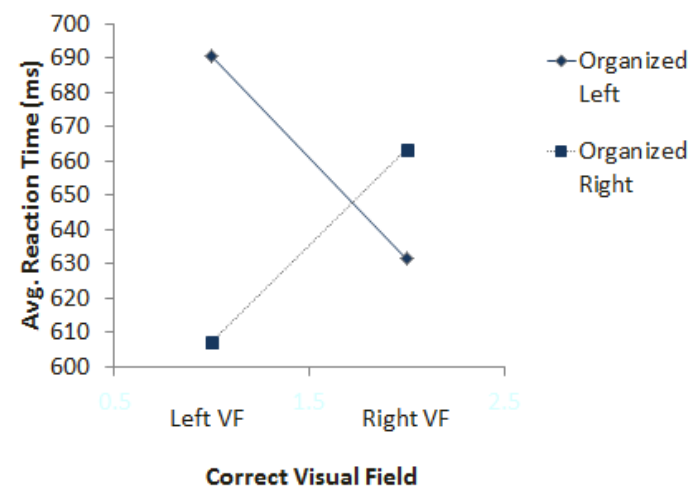

Figure 3: Correct Average Reaction Times (Correct Visual Field x Organized Field)

Figure 3 shows the correct average reaction times when compared in a $2 \times 2$ interaction between Correct Visual Field and Organization, $F(1,39)=37.044, . p=.01, \eta^{2}=.529$. In the legend, Organized Right and Organized Left indicate which visual field the dot clusters were located in. On the $\mathrm{x}$ axis, Left VF and Right VF indicate which visual field the correct response was in. (See table 3.1 in the appendix for means and standard errors).

Average reaction time data was analyzed with a repeated measures test and significance was found between visual field and organization. Based on Figure 3, the hypothesis that reaction time would be faster in the right visual field when dot clusters were unorganized was supported, but there was no support that the left visual field would have a faster reaction time to organized clusters of dots. In summary of this significant interaction, disorganization in dot clusters led to faster reaction times in both visual fields.

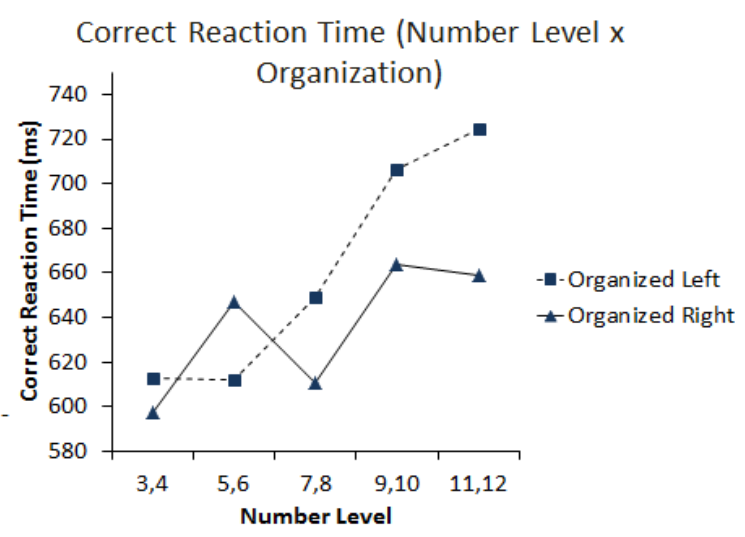

Figure 4: Correct Average Reaction Times Repeated Measures Interaction (Number Level x Organization). This figure shows how the average correct reaction times changed as the number level of the dot clusters were increased.

The results contained in Figure 4 show that when dot clusters were organized in the left visual field, reaction time steadily increased as the number level increased (increase in dots). However, when dot clusters were organized in the right visual field, reaction time did not show a steady increase with number level. The hypothesis that reaction time would increase with number level was only supported when dots were organized in the left visual field. The right visual field progression and possible explanations are explored in the discussion section.

\section{Discussion}

The current experiment was derived from previous research that indicated the left hemisphere (right visual field) was expected to use a counting ability to assess unorganized clusters of dots most efficiently with respect to both accuracy and reaction time (Nieder \& Dehaene, 2009). The right hemisphere (left visual field) was expected to use an estimation ability to assess clusters of organized dots most efficiently with respect to both accuracy and reaction time (Piazza, Mechelli, Price, \& Butterworth, 2006). The left hemisphere (right visual field) showed the highest accuracy when participants judged unorganized clusters of dots in the third number level (dots of 7 and 8), which showed support for the counting ability of the left hemisphere. The data also revealed that unorganized clusters of dots presented in the right visual field yielded an overall faster reaction time in the left hemisphere. This showed support for the hypothesis that reaction time would be be faster in the right visual field when dot clusters were unorganized. The reaction time data for the right visual field supports that the left hemisphere's counting ability responds more quickly to disorganization in clusters than when compared to organization. The accuracy data for the right visual field shows that the counting ability of the left hemisphere is most accurate when clusters were disorganized in the third number level ( 7 and 8 dots) in comparison to the right hemisphere's estimation ability. This supported that disorganization in the right visual field induced a more accurate response than organization in the right visual field, 
which directly supported the counting ability of the left hemisphere.

The data showed fewer areas of support for the right hemisphere (left visual field). The left visual field was not as accurate in the any of the five number levels as the right visual field even when the dot clusters were organized in structure. Organized structure had initially been predicted to make the left visual field more accurate but the data did not support this claim, which in turn did not support the estimation claim of the right hemisphere. The data from correct average reaction times indicated that organized clusters displayed in the left visual field slowed reaction time when compared to unorganized clusters shown in the left visual field. The hypothesis that organized clusters would yield a faster reaction time in the left visual field was also not supported by the data. The lack of support for these hypotheses led to the conclusion that either the estimation ability is not present in the right hemisphere in the third number level, or it functions in a way that was not accurately tested through the current research.

What these data implicate overall is that smaller dot clusters before the third number level (dots 7 and 8 ) of both visual fields are similarly accurate in number judgment regardless of organization. This implies that both estimation and counting are effective at small number levels. Data from the third number level show a significant separation where organization in the right visual field causes a very low accuracy while disorganization causes a very high accuracy. This result indicates that the counting ability indeed responds more to unorganized clusters in the right visual field. The left visual field was very similar in accuracy in the third number level for both organized and disorganized clusters. The accuracy values for the left visual field were between the two extreme values of the right visual field. This led to the idea that estimation was a less efficient process for the right hemisphere in the third number level while the counting process of the left hemisphere was more efficient. The reaction time data implicate that organized structures slow the average reaction time. This indicates that reaction time is faster when disorganized clusters are presented to both visual fields.

The results obtained support few predictions that were made for this study based on the previous research. One supported prediction was that as the number of objects being estimated increased, the average reaction time would decrease. An almost linear transitional increase can be noted in the left visual field when the dot clusters were organized and the number level increased. This linear increase supported the prediction that the reaction time, when using the estimation ability of the right hemisphere, decreased as the number level increased.

One limitation of this study was that the size of dots presented was not based on standard value. A current study indicated that dot size, dot grouping, and dot spacing may cause the counting and estimation abilities of the left and right visual fields to be affected in either a positive or negative manned based on the type of orientation, size, and spacing of dot clusters (Koesling et al., 2004). Another limitation of the current study was that excess movement and shifting of the participants was not effectively controlled. One aspect that the study relied on was the distance from the participant to the computer screen. Every participant began the study $57 \mathrm{~cm}$ away from the screen, but not all participants remained still during the experiment which does not guarantee that every participant finished the experiment $57 \mathrm{~cm}$ away from the screen. Shifting away from the desired distance could have easily had detrimental effects on the data. A more restrictive setting could have ensured less movement from participants. One final problem with the current study was the fact that three different organized structures of dots were used, but only one disorganized structure was used. The data would be more reliable if only one organized structure and one disorganized structure were used for every number level.

One of the strengths of this study was the abundant amount of number levels tested. The data from each of the 5 number levels shows the full spectrum of the estimation and counting abilities of the visual fields in respect to reaction time and accuracy. The trends in each visual field can easily be traced until estimation and counting abilities each become too closely similar and have no significant interactions (number levels 4 and 5) because of the large number of dots shown in the short amount of time given. A different strength of the current study was the counterbalancing of organized and disorganized dot clusters. Each participant viewed the same number of both types of organized and disorganized structure. This ensured that the datum from each participant was balanced in organized and disorganized structures. If the slide presentations had not been counterbalanced with the two types of structures, the data would have been useless.

The current study could lead to future studies by relating the spacing and size of dots to accuracy and reaction time. If a paradigm for spacing and size of dots could be developed, the current study could be improved. Another future study that could be developed from this study would be to limit interaction of the two visual fields. The current study relied on participants to keep their eyes focused on the center of the screen; a more efficient experiment would clearly divide the visual fields so that no interaction would occur. If the left and right visual fields were separated by an object or divider, this would ensure that only one visual field could assess each cluster at a time. One possible study that could compare estimation and counting abilities would be to explore different display times of both the target slides, dot presentation slides, and the response slide. A study of this type could create a paradigm that may identify a specific length of time for both visual fields to view clusters so that they are most accurate in judgment. A recent study also challenges the idea of left hemisphere versus right hemisphere completely (Borst, G., Thompson, W. L., \& Kosslyn, S. M. 2011). The concept developed in this study supports a top - down division (dorsal - ventral) of the processes in the brain. More research in the future could also enhance the data from this new study especially if the data could be replicated.

Much research still needs to be completed in the area of brain lateralization and numerical processes. From the data acquired from this study and from past research, disorganization of dot structures yields a faster reaction time in both visual fields, which supports the counting ability of the left hemisphere. Accuracy data did not support the estimation ability of the right hemisphere but did show support for the counting ability of the left hemisphere. Reaction time data showed a steady increase with number level in the right visual field when dots were organized in structure. This indicated that the estimation ability of the right 
hemisphere showed a linear increase in reaction time as the number level was raised. These data indicate that perhaps the areas of the brain that contain numerical ability may not reside solely in the left and right hemispheres, but instead may still be unknown.

\section{References}

American Psychological Association. (2002). American psychological association ethical principles of psychologists and code of conduct. Retrieved from http://www.apa.org/ethics/code2002.html

Bertletti, I., Lucangeli, D., Piazza, M., Dehaene, S., \& Zorzi, M. (2010). Numerical estimation in preschoolers. Developmental Psychology,46(2),545-551.

Borst, G., Thompson, W. L., \& Kosslyn, S. M. (2011, June 27). Understanding the dorsal and ventral systems of the human cerebral cortex: beyond dichotomies. American Psychologist. Advance Online Publication. doi: 10.1037/a0024038

Burr, D.C., Turi, M., \& Anobile, G. (2010). Subitizing but not estimation of numerosity requires attentional resources. Journal of Vision, 6(10),1-10.

Dehaene, S., \& Changeux, J. (1993). Development of elementary numerical abilities: a neuronal model. Journal of Cognitive Neuroscience, 5(4), Retrieved from http://www.unicog.org/publications/DehaeneChangeux_ NeuronalModelNumbers_JOCN1993.pdf

Frith, C., \& Frith, U. (1972). The solitaire illusion. Perception \& Psychosis, 11(6), 409-410. doi: 10.3758/BF03206279

He, L., Zhang. J., Zhou, T., \& Chen, L. (2009).

Connectedness affects dot numerosity judgment: implications for configural processing. The Psychonomic Society, Inc., 16(3), 509-517. doi: 10.3758/PBR.16.3.509

Koesling, H., Carbone, E., Pomplun, M., Sichelschmidt, L., \& Ritter, H. (2004). When more seems less - non-spatial clustering in numerosity estimation. Paper presented at the Early Cognitive Vision Workshop, Isle of Skye, UK. Retrieved

fromhttp://umboston.academia.edu/MarcPomplun/Papers 1571369/When_More_Seems_Less_NonSpatial_Clustering_in_Numerosity_Estimation

Nieder, A., \& Dehaene, S. (2009). Representation of number in the brain. Annual Review of Neuroscience,32, 185208. doi: 10.1146/annurev.neuro.051508.135550

Piazza, M. (2010). Neurocognitive start-up tools for symbolic number representations. Space, Time, and Number, 14(12), 542-551. doi: 10.1016/j.tics.2010.09.008

Piazza, M., \& Dehaene, S. (2004). From number neurons to mental arithmetic: the cognitive neuroscience of number sense. Cognitive Neurosciences $3^{\text {rd }}$ Edition, 856-875. Retrieved from http://www.scribd.com/doc/16531938/From-NumberNeurons-to-Mental-Arithmetic-The-CognitiveNeuroscience-of-Number-Sense-Piazza-Dehaene-2004

Piazza, M., Mechelli, A, Price, C.J., \& Butterworth, B. (2006). Exact and approximate judgments of visual and auditory numerosity: an fMRI study. In Press: Brain Research, Retrieved from http://www.mathematicalbrain.com/pdf/2006PIAZZET AL.PDF
Ratinckx, E., Brysbaert, M., \& Reynvoet, B. (2001). Bilateral field interactions and hemispheric asymmetry in number comparison. Neuropsychologia,39(4), 335-345.

Retrieved from http://biblio.ugent.be/input/download?func=downloadFil e \&fileOId=599019

Siegler, R., \& Booth, J. (2004). Development of numerical estimation in young children. Child Development, 75(2), 428-444. Retrieved from http://www.psy.cmu.edu/ siegler/sieglerbooth-cd04.pdf

Siegler, R., \& Opfer, J. (2003). The development of numerical estimation: evidence for multiple representation of numerical quantity. Psychological Science, 14(3), 237243. doi: 10.1037/a0017887 